Paper to be presented at the XXXVth ASTIN Colloquium, Bergen, 6 - 9th June 2004

\title{
Pragmatic Insurance Option Pricing
}

\author{
by Jon Holtan \\ If P\&C Insurance Company Ltd \\ Oslo, Norway \\ Email: jon.holtan@if.no \\ Telephone: +4792260065
}

\begin{abstract}
This paper deals with theoretical and practical pricing of non-life insurance contracts within a financial option pricing context. The market based assumption approach of the option context fits well into the practical nature of non-life insurance pricing and valuation. Basic facts in most insurance markets like the existence of quite different insurer price offers on the same claims risk in the same market, support the need for this approach. The paper outlines insurance and option pricing in a parallel setup. First it takes a complete market approach, focusing dynamic hedging, no-arbitrage and risk-neutral martingale valuation principles within insurance and options. Secondly it takes an incomplete market view by introducing supply and demand effects via purchasing preferences in the market. Finally the paper discusses pragmatic insurance price models, parameter estimation techniques and international best practice of insurance pricing. The overall aim of the paper is to describe and unite the headlines of the more or less common insurance and option price theory, and hence increase the pragmatic understanding of this theory from a business point of view.
\end{abstract}

\section{Keywords}

Insurance and financial option contracts; insurance and option pricing theory; complete and incomplete markets; dynamic hedging and no-arbitrage; risk-neutral martingales; purchasing preferences; risk, cost and market price adjustments; parameter estimation; pragmatic and best practice pricing. 


\section{Introduction}

There has been a considerable attention on the relationship between insurance and financial pricing theory the last two decades after the breakthrough establishment of the financial martingale theory made by Harrison and Kreps (1979) and Harrison and Pliska (1981). There also seems to have been an increasingly development speed in the 1990-thies after the important works on dynamic pricing of arbitrage-free (re)insurance markets made by Delbaen and Haezendonck (1989) and Sondermann (1991); see Embrechts (2000) for an exiting overview description. In this paper we will try to describe and unite the headlines of this common theory to a practical understanding and practise in non-life insurance pricing, and thus also try to address some future theoretical challenges. A direct comparison of insurance pricing versus option pricing will also give deeper insight to the relationship between the two pricing techniques, and hence make the bridge between the financial and the actuarial approach to pricing a bit wider, - not only for academics on the universities, but also for us practical working actuaries in the insurance business industry.

The paper is organized as follows. Section 2 addresses some actual pricing questions and problems. Section 3 outlines characteristics of insurance contracts within an option pricing context. Section 4 outlines and discusses dynamic hedging, no-arbitrage and put-call parities in a parallel option and insurance approach. Section 5 puts the pricing challenges into a risk-neutral martingale pricing approach. Section 6 discusses effects generated by incomplete markets and introduces a purchasing preference element to insurance pricing. Section 7 introduces some pragmatic insurance price models. Section 8 discusses relevant parameter estimation techniques within insurance pricing. Section 9 presents and discusses international best insurance pricing practices. Section 10 gives some concluding remarks.

\section{Pricing non-life insurance contracts versus financial options}

The common basic idea of both financial options and insurance contracts is to transfer an economical risk from one part to another against a specific payment. To do so the buyer and the seller of the contract need to agree upon a price before the contract begins to run. A common used market methodology approach is to split this pricing into one technical valuation part and one part purely influenced by supply and demand mechanisms. Hence the point is first to find the technical fair values of the contracts, and then adjust these values to real sales prices based on purchasing preferences or dispositions in the market. Finn and Lane (1997) supports this view by saying: "There are no right price of insurance; there is simply the transacted market price which is high enough to bring forth sellers, and low enough to induce buyers". This statement definitely rules also in the financial derivate market. Hence, quite distinct speaking, the price of an option or insurance contract is the premium one pays for it, while the value is what it is worth. 
The difference between value and price/premium depends very much on the market conditions of the option or insurance contract. We have to behave some main market differences:

Insurance market:

- Most often standard one-year contracts

- Most often no real efficient market place for buying and selling contracts

- Not only price, but also product and service quality are important

- Price per contract depends on and varies between the contract buyers

Financial option market:

- Many different types of contracts with no standard time condition

- Most often an efficient market place for buying and selling contracts

- Only price is important to most customers

- Price per contract does not depend on the buyers of the contracts

The more or less complete market conditions of financial options generate a smaller difference between value and price/premium than the more incomplete insurance market. Hence, particularly for insurance contracts the theoretical challenge is to include not only the valuation part of pricing, but also the supply/demand part into the price models. Some interesting key questions within this approach are:

- Why do different insurance players offer different prices for unique risks? and under what conditions will more unique market prices for unique risks be generated?

- What is the optimal price per risk in a complete insurance market? - and in an incomplete market?

In this paper we will stress these questions by first focusing the valuation part of pricing in a complete market setup, and then include also incomplete market based purchasing preferences. Equivalent questions are well known and handled within financial option pricing theory. Hence the market model context of option pricing fits well into the practical nature of non-life insurance pricing. To get more hands on pricing similarities and differences let us therefore outline insurance and option pricing in a parallel approach.

\section{Insurance contracts in a financial option context}

An option is a contract giving the holder a right to buy or sell an underlying object at a predefined price during a predefined period of time. An option condition is that the underlying object has an uncertain stochastic future value. Financial stocks are the most common option object. However, the claims risk of an insurance customer may be interpreted as another option object. Hence we may define a non-life insurance contract as an option. We have the following correspondingly definitions: 
Call option on stocks: The holder of a call option gives the right, but not the obligation, to buy a stock at a predetermined date (maturity time) and price (strike price).

Insurance contract: The holder of an insurance contract gives the right to get covered all incurred insurance claims within a predetermined date (maturity time) and at a predetermined price (the deductible or excess point).

Assume two different underlying risk processes $S(t)$ and $X(t)$, where $S(t)$ is the stock price process up to time $t$ and $X(t)$ is the accumulated insurance claim process up to time $t$. That is, assume $X(t)=\sum_{i=1}^{N(t)} Y_{i}$, where $N(t)$ is the number of incurred claims up to time $t$ and the $Y_{i}$-ies are the claims severities.

Let $C$ be a European call option contract on the stock price process $S(t)$ and $Z$ and $Z^{*}$ two different insurance contracts on the claims risk process $X(t)$. Assume time $t=0$ as the start time of all contracts and time $t=T$ as the maturity time. Hence let:

$C(T)=\max (S(T)-K, 0)$, where $C(T)=$ call option payment value at time $T$, $S(T)=$ stock price at time $T$ and $K=$ strike price.

$Z(T)=\sum_{i=1}^{N(T)} \max \left[Y_{i}-D, 0\right]$, where $Z(T)=$ sum insurance payment value at time $T, N(T)=$ number of claims up to time $T, Y_{i}=$ incurred claim amount of claim number $i$ up to time $T$ and $D=$ deductible for each claim occurrence.

$Z *(T)=\max \left[X(T)-D^{*}, 0\right]$, where $Z *(T)$ is the sum insurance payment value at time $T, X(T)=$ accumulated claim sizes at time $T$, and $D^{*}=$ deductible or excess point of $X(T)$.

Hence $Z$ is an excess-of-loss insurance contract and $Z^{*}$ is a stop-loss insurance contract. We also observe that $\mathrm{Z}$ may be interpreted as a stochastic sum of $N$ single European call options and $Z^{*}$ as an ordinary European call option. Hence within this context we may name these insurance contracts as insurance call contracts, and the pricing of them as insurance option pricing.

The crucial difference between stock option contracts and insurance contracts is generated by the underlying stochastic processes of the contracts. The future values of a stock may occur up and down from a spot value at time $t=0$, while the accumulated insurance claim values only will increase from a start value equal zero at time $t=0$. These differences are reflected in the classical stochastic assumptions of the processes where the variation of $S(t)$ assumes to follow a geometric Brownian motion process and $X(t)$ most often assumes to be a compound homogeneous Poisson process where the claim number process $N(t)$ is a homogeneous Poisson process (with claims frequency parameter $\lambda$ ) and the 
claims severities $Y_{i}$-ies are independent and identically distributed random variables and independent of the counting process $N(t)$.

The assumption of a geometric Brownian motion process for $S(t)$ - where the running time increments of the variation of the process follow a normal distribution function - is based on the idea that the spot price of a stock at time $t$ already reflects the future expected value of the stock. Hence all relevant information influencing the future expected return on the asset is assumed continuously build into the running value (the spot price) of the asset, that is, we assume a complete stock market where efficient market trading of the assets generate these characteristics.

Even if the geometric Brownian motion process itself is not very simple, we may say that the effects of the model assumptions of the stock price process generate quite simple and nice pricing challenges within the financial option markets. The compound homogeneous (or even more realistic, a mixed (heterogeneous)) Poisson process assumption of the claims risk makes on the other hand more intricate problems to handle within a realistic insurance market. The crucial purpose of pricing the option and insurance contracts is in any case to find explicit expressions of the current value of the contracts at the contractual start time, that is, in this setup at time $t=0$. Or even more general we may want to find explicit expressions of the current values of the contracts $C, Z$ and $Z^{*}$ at any time $t(<T)$ before the maturity time $T$.

\section{Dynamic hedging, no-arbitrage and put-call parities}

Following the original - and now Nobel famous - development lines made by Black and Scholes (1973) and Merton (1973), the explicit expressions of $C, Z$ and $Z^{*}$ should be based on dynamic hedging of the underlying stochastic portfolios. The key basis of the Black-Scholes formula of financial option values is to create risk free synthetic portfolios by continuously (dynamic) purchasing (hedging) a certain share of the underlying asset and a certain amount of a risk less bond. The hedging strategy has two key properties: 1) It replicates the payoff of the option, and 2) It has a fixed and known total cost. Hence the value of the option at any time $t$ may be explicitly expressed by the combination of the shares of the asset and the bond.

The most important assumption behind this dynamic hedging based pricing theory is the no-arbitrage assumption. That is, we assume no opportunities to make risk less profits through buying and selling financial security contracts. This assumption generates the existence of a put-call parity, that is, a fundamental relationship between the values of a call option and a put option (the right to sell a security to a strike price). Let $\tilde{C}$ be an European put option with the same strike $K$ and maturity $T$ as the call option $C$ :

$\tilde{C}(T)=\max [K-S(T), 0]$ where $\tilde{C}(T)=$ put option value at maturity time $T$, $S(T)=$ stock price at time $T$ and $K=$ strike price. 
Then we have the basic put-call parity known from every basic option text book:

$$
C(t)-\tilde{C}(t)=S(t)-e^{-r T} K
$$

where $r$ is the risk free rate of interest. In words: the value of the call minus the value of the put is equal to the value of the stock minus the present value of a risk less bond $K$. Any deviation from the put-call parity does constitute an arbitrage opportunity because simultaneously selling and buying the put-call portfolio (left hand side of (1)) and the stock-bond portfolio (right hand side of (1)) yield a risk less profit equal the difference between the values of the portfolios. Hence within option price theory the no-arbitrage assumption is equivalent to the existence of the put-call parity. In fact, a more far-reaching consequence of the no-arbitrage assumption is that two identical financial risks must have the same value (the law of one price).

Turning to the insurance contracts, we may also construct put-call parities for the excess-of-loss and stop-loss contract. This depends however on the existence of so-called insurance put contracts. Hence let $\tilde{Z}(T)$ and $\tilde{Z} *(T)$ respectively be the values at maturity time $T$ of an excess-of-loss put contract and a stop-loss put contract. We then have:

$$
\begin{gathered}
\tilde{Z}(T)=\sum_{i=1}^{N(T)} \max \left[D-Y_{i}, 0\right], \\
\tilde{Z} *(T)=\max \left[D^{*}-X(T), 0\right] .
\end{gathered}
$$

The practical interpretation of the insurance put contract is that the holder of the contract gives the right to a payment of $D$ or $D^{*}$ against a self-financing of the claims $X(T)$ during the period $(0, T)$. This is obviously a right the holder of the contract only will use if the incurred claims are less than $D$ or $D^{*}$. Hence the owner of a claims risk could by buying an insurance call contract and at the same time sell an insurance put contract on the same underlying risk gain a risk-free cash flow equal to the deductible $D$ or $D^{*}$. The claims occurrence during the contract period will not influence this cash flow.

By use of simple algebra we find straightforward the general put-call parity expressions of the insurance contracts:

$$
\begin{aligned}
& Z(T)-\tilde{Z}(T)=X(T)-D(T), \text { where } D(T)=N(T) D \\
& Z^{*}(T)-\tilde{Z}^{*}(T)=X(T)-D^{*}(T), \text { where } D^{*}(T)=D^{*}
\end{aligned}
$$

That is, the value of the call minus the value of the put is equal to the value of the accumulated claim amounts minus the (present) value of $D(T)$ of each contract. Hence the structure of the parities are identical to the financial option parity. 
Following the same arguments as for option contracts, any deviation from the putcall parity does constitute an arbitrage opportunity in the insurance market because simultaneously buying and selling the insurance put-call portfolio (left hand side of (4) and (5)) and the claims-bond portfolio (right hand side of (4) and (5)) yield a risk less profit equal the difference between the values of the portfolios. It is however important that these relationships depend on the existence of an insurance put contract $\tilde{Z}$ or $\tilde{Z} *$ and the existence of an efficient market place of buying and selling insurance calls and puts.

But what about dynamic hedging of insurance contracts? The existence of insurance put-call parities and no-arbitrage in an insurance market are important elements, but not sufficient to replicate a risk free payoff of an insurance risk by a hedging strategy. It is well known that the ordinary insurance claims risk process $X(t)$ is difficult to directly hedge away by dynamic trading, and by this follow the classical financial pricing theory. Delbaen and Haezendonck (1989), however, assumed that an insurer at any time $t$ can sell the remaining claim payments $X(T)-X(t)$ over the period $(t, T)$ for some premium $p(T-t)$. Hence the value of the portfolio of claims risks at time $t$ is $I(t)=X(t)+p(T-t)$, where $I(0)=p(T)$ and $I(T)=X(T)$. This assumption of dynamic buying and selling insurance risks during the insurance period makes the crucial possibility for dynamic hedging also in insurance markets.

By following the Black-Scholes approach, a straightforward description of this risk less insurance hedge at time $t$ is: The value of the insurance contract at any time $t$ may be expressed by a combination of a share $\Delta_{1}(t)$ of the underlying claims risk portfolio $I(t)$ and an amount of a risk less zero-coupon bond $B_{1}(t)$ maturing at time $T$. Hence the value of the insurance hedge portfolio at time $t$ is

$$
\Delta_{1}(t) I(t)-e^{-r(T-t)} B_{1}(t)
$$

Hereby the dynamic hedging of $I(t)$ aim at continuously rebalance (as for options we assume that rebalancing costs do not include transaction costs) the hedge by holding $\Delta_{1}(t)$ shares of $I(t)$ long and a bond maturing at time $T$ to $B_{1}(t)$ short.

Correspondingly, the value of the option hedge portfolio at time $t$ is

$$
\Delta_{2}(t) S(t)-e^{-r(T-t)} B_{2}(t),
$$

where the share $\Delta_{2}(t)$ - the delta of the option - is the rate of change of the value of the option with respect to the underlying value of the stock, that is, the relative amount the option value $C(t)$ will change when $S(t)$ change. Hence, $\Delta_{2}(t)$ is close to 1 when the option is "deep in the money" and near expiration (high probability to expire in the money), and close to 0 if the option is "deep out of money" and near expiration (high probability to expire out of the money). 
We may correspondingly interpret $\Delta_{1}(t)$ of $I(t)$ as the relative amount the insurance contract value $Z(t)$ will change when $I(t)$ change. What this interpretation really means is not obvious. The future values of a stock may increase and decrease with no limits (but not below zero) from a running spot value at time $t$. These increase and decrease are also true for $I(t)$, but there is a lower limit of $I(t)$ equal $X(t)$. That is, at time $t$ the future value of $I(t)$ can not be lower than $X(t)$ because $I(t)=X(t)+p(T-t)$ and $X(t)$ can not decrease. Hence, in that moment $X(t)$ exceeds the deductible $D$ or $D^{*}$ the insurance contract will be - and stay - "in the money", that is, $Z(T)>0$ or $Z^{*}(T)>0$. Because $I(t)$ consists of both $X(t)$ and $p(T-t)$ there is - in contrast to the option and $S(t)$ no one-to-one relationship between the values of $I(t)$ and $Z(t)$ even if $Z(t)$ will certainly stay "in the money" the rest of the period $(t, T)$.

Correspondingly to Black-Scholes, the value of the insurance hedge portfolio is in fact equal to the value of the insurance contract. Particularly we have

$$
\begin{aligned}
Z(0)= & \text { Value of insurance contract at time } t=0 \\
& =\text { Value of insurance hedge portfolio at time } t=0 \\
& =\Delta_{1}(0) I(0)-e^{-r T} B_{1}(0)=\Delta_{1}(0) p(T)-e^{-r T} B_{1}(0) .
\end{aligned}
$$

That is, the value of the insurance contract at time $t=0$ is equal to a linear functional $\Delta_{1}$ of the premium $p(T)$ of the risk period $(0, T)$ minus the present value of the bond $B_{1}$ (which should be interpreted as the amount needed to pay deductibles in $(0, T))$. The most efficient mathematical machinery to find more explicit expressions of $\Delta_{1}$ and $B_{1}$, and hence value expressions of $Z$ and $Z *$, is to use the now classical martingale approach under the risk-neutral valuation principle.

\section{Risk-neutral martingale pricing}

The dynamic hedging strategy and the no-arbitrage assumption are closely related to the risk-neutral valuation principle. This principle states that in a risk-neutral world all assets have an expected rate of return equal to the risk-free rate of return. In the real world we have to consider the relationship between risk (volatility) and reward (expected return) to price an asset. In a risk-neutral world, reward has been normalized through the no-arbitrage mechanisms, that is, all relevant market information are assumed known and build into the pricing models so that no investors will expect a higher reward than the risk-free rate of return. This information transaction is technically given by tuning the classical real world probability measure $P$ on the probability space $(\Omega, F, P)$ into a filtered uniquely equivalent probability measure $Q$ (by use of the so-called Girsanov transformation) on the probability space $\left(\Omega, F,\left(F_{t}\right)_{t \geq 0}, Q\right)$ where $F_{t}$ can be viewed as the market information available at time $t$. Hence we make expected value calculations based on $Q$ instead of $P$. This information transaction is assumed valid at every time $t$, and hence the underlying risk process (with respect to 
measure $Q$ ) turns out to be a martingale process where at each time $t$ the expected next value direction of the process is zero (corrected for the risk-free rate of return). In practice this implies that it is impossible to earn (risk less) money by studying the history of a stock, that is, all relevant and available information about the stock and the market are assumed fully integrated into the running spot price of the stock. The technical link - called the Fundamental Theorem of Asset Pricing - between the no-arbitrage assumption and the martingale process was the key discovery made by Harrison and Kreps (1979) and further developed by Harrison and Pliska (1981) and generalized by Delbaen and Schachermayer (1994). See Delbaen and Schachermayer (1997) for a rigorous mathematical summary of relevant main results.

The setup by Delbaen and Haezendonck (1989) of the insurance claim risk portfolio $I(t)=X(t)+p(T-t)$ with efficient dynamic hedging possibilities, generates an arbitrage-free insurance market. Hence this specific market setup turns into be risk information transformed, that is, from only assuming the original probability distribution of the insurance risk process $X(t)$ (the classical static actuarial approach) to instead assume a filtered distribution of $X(t)$, in this case, give more weight to unfavourable events of $X(t)$. Again this approach is technically given by tuning the classical real world probability measure $P$ into a equivalent filtered risk neutral probability measure $Q$. Hence we make expected value calculations on the portfolio value process $I(t)$ by using the measure $Q$ instead of directly on the risk process $X(t)$ by using the measure $P$. The portfolio value process $I(t)$ is hence called a $Q$-martingale, i.e. at each time $t$ the expected next value direction of the process $I(t)$ is zero (corrected for the risk-free rate of return). Within this model a practical interpretation is that it is impossible to earn money by studying the claims history of $(0, t)$ of the insurance portfolio $I(t)$ because all relevant information about the insurance risks are assumed fully reflected into the running risk premium $p(T-t)$ at time $t$.

To sum up: While the geometrical Brownian motion assumption of the variation of the stock value $S(t)$ directly generates the martingale process of $S(t)$, we have to transform the accumulated Poisson claims risk process $X(t)$ into a claims risk portfolio $I(t)$ consisting of both historical claims and premium for future claims to generate a martingale process indirectly. Embrechts et al (1999) gives a thorough introduction to this link between stochastic processes in insurance and finance.

Still following the approach of Delbaen and Haezendonck (1989) and given our claims risk process assumptions in chapter 3 , the fair values at time $t$ of the excess-of-loss contract $Z$ and the stop-loss contract $Z *$ become

$Z(t)=E_{Q}\left[e^{-r(T-t)} Z(T)\right]$, where $Q=$ adjusted risk-free probability measure, $\left.Z^{*}(t)=E_{Q^{*}} \mid e^{-r(T-t)} Z^{*}(T)\right\rfloor$, where $Q^{*}=$ adjusted risk-free probability measure,

while the correspondingly fair value at time $t$ of the call contract becomes 
$C(t)=E_{Q^{* *}}\left[e^{-r(T-t)} C(T)\right]$, where $Q^{* *}=$ adjusted risk-free probability measure.

Hence the fair values of the contracts at time $t=0$ are

$$
\begin{aligned}
& \begin{aligned}
& Z(0)=E_{Q}\left[e^{-r T} Z(T)\right] \\
& \begin{aligned}
Z^{*}(0) & =E_{Q^{*}}\left\lfloor e^{-r T} Z^{*}(T)\right\rfloor=e^{-r T}\left\lfloor E_{Q^{*}} X(T)-E_{Q^{*}} D\right\rfloor \\
& =e^{-r T}\left\lfloor E_{Q^{*}} N(T) E_{Q^{*}} Y-E_{Q^{*}} D\right\rfloor
\end{aligned} \\
& C(0)=E_{Q^{* *}}\left\lfloor e^{-r T} C(T)\right\rfloor=e^{-r T}\left\lfloor E_{Q^{* *}} S(T)-E_{Q^{* *}} K\right\rfloor
\end{aligned}
\end{aligned}
$$

Correspondingly, the straightforward derived Black-Scholes formula of $C(t)$ turns out to be

$$
C(t)=N\left(d_{1}(t)\right) S(t)-e^{-r(T-t)} K N\left(d_{2}(t)\right),
$$

where $N(\cdot)$ = cumulative normal distribution function and $d_{1}(t)$ and $d_{2}(t)$ are given in most basic text books of option theory and of course in Black and Scholes (1973). Hence $\Delta_{2}(t)=N\left(d_{1}(t)\right)$ and $B_{2}(t)=K N\left(d_{2}(t)\right)$. We also observe that the martingale filtered measure $Q^{* *}$ of (11) reflects the values of $\Delta_{2}(t)$ and $B_{2}(t)$ in (12), that is,

$$
\begin{gathered}
E_{Q^{* *}} S(T)=N\left(d_{1}(t)\right) S(t)=\Delta_{2}(t) S(t), \\
\text { and } E_{Q^{* *}} K=N\left(d_{2}(t)\right) K=B_{2}(t) .
\end{gathered}
$$

Hence $N\left(d_{1}(t)\right)$ is the rate of change at time $t$ of the value of the call with respect to changes in stock price $S(t)$, while $N\left(d_{2}(t)\right)$ should be interpreted as the probability that $C(t)$ at the maturity time $T$ will be "in the money".

When studying (9), (10) and (11) we observe the structural similarities between the fair value expression of the insurances $Z$ and $Z^{*}$ and the option $C$. In fact the values are linear functions defined on a set of marketable assets. Quite general we have:

Financial call option

The value at time $t$ increases...

- the longer time to maturity,

- the lower the strike price is,

- the higher the spot price is*,

- the higher the risk free rate is,

- the higher the stock volatility is.

\section{$\underline{\text { Insurance contract }}$}

The value at time $t$ increases...

- the longer time to maturity,

- the lower the deductible is,

- the higher the claims have been*,

- the higher the risk free rate is,

- the higher the claims risk is. 
Four out of five parameters are easy to understand. However, one $*$-comment on the spot price versus the claims value at time $t$ is needed: At any time $t$ the spot value of a stock is a positive value, while the insurance claims have a zero start value at time $t=0$ and possibly a positive value at any time $t>0$. Hence the expression "the higher the claims have been" is related to the claims occurred up to time $t$.

The great practical usefulness of the Black-Scholes option formula is due to that only the stock volatility is unknown as valuation parameter at the valuation time. Option dealers are hence often just called volatility dealers, where implied volatility or tight market knowledge is often used as the parameter estimation technique of the volatility. The fact that the value of an option depends on the volatility of the underlying but not on its expected return (which is in accordance to "risk-neutrality"), numbers as one of the great insights from the Black-Scholes formula. Criss (1997) is hereby a pedagogical reference as a wide and basic introduction to financial options and option pricing.

As for options the claims risk is - so far and within the above setup - the most stochastic based parameter to estimate for insurance contracts. However to generate more specific expressions of (9) and (10) we have to deal with the filtered probability measure $Q$. Delbaen and Haezendonck (1989) - see also Embrechts (2000) - outlines that certain assumptions and conditions of the filtered $Q$-measure lead to well-known premium principles identical to the expected value principle, the variance principle and the Esscher principle, all generating different safety loading factors in addition to the pure expected claims risk up to time $T$ based on the classical probability measure $P$. Hence the choice of the technical properties of $Q$ turns out to be the essential part of the valuation of $Z$ and $Z^{*}$, or in other words, it all depends on what relevant extra information we put into the measure $Q$ at time $t$. The three premium principles are for instance based on the $a$ priori choice of safety loading as the (only) critical information bias. The safety loading is basically important, and corresponds to the need for ruin safety for the insurer and to the expected utility willingness of the customers to pay higher premium than the pure risk premium. However, other highly relevant information to include a priori in the price models and hence affecting the $Q$-measure are e.g. information about the administration and reassurance expensive and the demand for investment return on the insurance portfolio. Technically this expanded information input will be handled by an expanded price model combined with an augment at time $t$ of the information filtration $F_{t}$ on $Q$.

In a complete market setup, with perfectly efficient buying and selling of insurance contracts, we conclude that pure risk and cost based premiums are the sufficient pricing tasks to handle for an insurance company. In such a market, where the market and price equilibrium rules, the winners of the market will be those companies which are able to deliver the best unbiased and anti selected risk prices, the lowest administration expensive and the most efficient reassurance and investment return demands. However, as we will discuss further on in chapter 6 and 7 , true market information like purchasing preferences and the insurers' price position in the market will be crucial information to take into account in an incomplete market setup. 


\section{Incomplete market effects and purchasing preferences}

The triangle assumptions of dynamic hedging, no-arbitrage and market completeness are mathematically very pleasant to assume when developing asset pricing models and formulas. Even if there are many offence on these idealistic assumptions, the relative realism and robustness of them in the financial markets seems nevertheless to be one of the main reasons for the revolution of financial mathematics the last decades. The market place of financial derivatives and insurance contracts are, however, normally quite different. The insurance markets are most often characterized by higher transaction costs, higher and differentiated risk aversion, less price rationality and sensitivity, less or no dynamic hedging, less decision speed and fewer players than financial markets. In addition the insurance market may be divided into a direct insurance market and a reinsurance market, where the above incomplete characteristics usually are more common in the direct market than in the reinsurance market.

However, the insurance internet market place, with effective and price sensitive shopping of insurance offers, may more and more generate less incomplete effects on the direct markets. In a future setting with an insurance market as efficient and complete as the financial derivate market, we may keep the complete market assumption approach of insurance pricing. Until so, if we take an average pragmatic approach on today's insurance pricing, we definitely have to leave the idealistic assumption of market completeness. However, the pricing mechanisms and result outcomes of complete market models give deep pricing insight, and are important to have in mind when dealing with expanded incomplete models.

Mathematical assumptions of incomplete markets generate much heavier theoretical models. A precise definition - given in Harrison and Pliska (1983) states that a market is complete if and only if there is only one equivalent martingale measure of the underlying stochastic process $S(t)$ (the stock market), or in our case, $I(t)$ (the insurance market). The practical interpretation of this definition should be that in complete markets there exists a unique price for each unique contractual risk - independent of the insurance players - and that each contract can be perfectly hedged or replicated in the market. Market assumptions which do not satisfy the price uniqueness per risk contract generate incomplete markets. Typically assumptions - see Embrechts and Meister (1997) - which generate incomplete markets are:

- Jumps in the underlying stochastic process with random size occur: For instance large jumps in stock markets because of bubble economy crash or in insurance markets because of heavy large-claim nature/weather catastrophes, or even simpler, an unexpected strong increase in the underlying market claims inflation.

- Stochastic volatility models, that is, assumptions of underlying stochastic processes with variable volatility along the time axis. For instance the variability of stocks or insurance claims which we assume to increase over the 
period of time; this may be realistic in e.g. some property insurance markets which have experienced increased whether variability the last years.

- Markets with transaction costs and/or investment constraints (so-called friction): For instance customers who loose some benefit build ups or have a higher product, service and/or brand preferences than pure price preferences, or different investment constraints between market players.

Some technical mathematical techniques have so far been developed to handle some problems of pricing derivatives in incomplete markets. Super replication (find the cheapest self-financing hedging strategy) and mean-variance hedging (find a trading strategy that reduces the actual risk of the derivative position) which is one of the so-called quadratic methods - are two examples; see e.g. Møller (2000) for more rigorous and detailed information and overviews on this subject.

The intuitive mathematical reason for the incompleteness of the above market assumptions is that in each case there are typically many different combinations of model parameters which influence the expanded model setup of the martingale process. Hence this property generates many different probability measures that turn the underlying process into many different so-called local martingales. A pragmatic consequence is that each financial or insurance contract (where each one of them is based on one unique financial or insurance risk) turns out to be valuable unique for each combination of buyers and sellers of the contract. We may hence conclude that the more incomplete an insurance market is, the more the prices of equivalent risks tend to vary in the market (the law of one price turned upside down). Remark that this connected statement assumes a deregulated insurance market with no price-cooperation between the insurer players in the market.

Another assumption which generates incomplete markets, at least within direct insurance, is to assume positive product, service and/or brand preferences of the buyers in addition to the price preference. That is, the buyers of the contracts have other "soft" purchasing preferences in addition to price when they act in the market. This assumption is highly realistic in most (or all) direct insurance markets, and probably one of the main drivers for the incompleteness of these markets. This market condition generates the introduction of purchasing rate as an important parameter within the price models. A definition of purchasing rate may be the likeability in the market to buy (or re-buy) a predefined insurance contract. This parameter varies typically individually between the insurance customers (just like their claims risks) and should normally be a function of the price sensitivity, the price position of the insurer and the more "soft" preferences like product-, service- and brand preferences. Hence, we say that buyers with less price sensitivity are more "soft" preferenced than other buyers, and vice versa. We conclude that this approach of individually purchasing rates - or probabilities matches the above local martingale interpretation of price uniqueness for each combination of insurance buyer and seller. Moreover by introducing the insurance purchasing preference the bridge to more sophisticated economical equilibrium 
price models is quite close; see e.g. Aase (1993) for an equilibrium analysis of dynamic reinsurance markets.

To sum up the main answers to the questions we stressed in chapter 2: Different insurance players offer different prices for unique risks mainly because each combination of buyers and sellers in incomplete markets generates individual unique contractual values. More unique market prices for unique risks will be generated in more complete markets with higher price sensitivity. Optimal price per risk in a complete insurance market is pure risk and cost price based, while the price in addition should be market adjusted in incomplete markets.

\section{Pragmatic insurance price models}

Corresponding to the individual martingale theory in chapter 6, we may put forward the following pragmatic price model approach of net insurance sales prices in the incomplete case:

Net sales price

$=$ pure risk price + internal cost price adjustments + external market price adjustments

Mathematically we may express the model as:

Net sales price $=$

$$
p_{i}(T)=M\left(C_{i}\left(Z_{i}\left(T \mid I_{r}\right) \mid I_{c}\right) \mid I_{m}\right)
$$

where

$$
\begin{aligned}
& p_{i}(t)=\text { net premium of risk contract } i \text { for period }(0, T) \\
& Z(\cdot)=\text { pure risk price pr risk contract } i \\
& C(\cdot)=\text { the cost price adjustment of the pure risk price } Z \\
& M(\cdot)=\text { the market price adjustment of the cost price } C \\
& F_{r}=\text { given claims risk information of }(0, T) \\
& F_{c}=\text { given cost information of }(0, T) \\
& F_{m}=\text { given market information of }(0, T) .
\end{aligned}
$$

The internal cost price adjustment $C(\cdot)$ in (13) is typically generated by specified goals of risk ratio (incurred claims in percent of earned premiums) and combined ratio (risk ratio + expense ratio (expenses in percent of earned premium)) for each relevant line of business. The risk ratio and combined ratio goals are usually part of the financial plan of the insurance company and based on opinions of necessary levels of risk loading, expenses, financial income, reassurance and demand for investment return of the company, all elements specifically broken down to each line of business.

The external market price adjustment $M(\cdot)$ in (13) is to some extent more difficult and more sophisticated to deal with than the pure risk and cost price elements. A 
possible definition of this price adjustments could be: All kinds of price deviation from the theoretical best known risk and cost based price. This definition supports the statement by Finn and Lane (1997) that there is no right price of insurance, but simply the transacted market price which is high enough to bring forth sellers, and low enough to induce buyers, ref chapter 2. Ref also our discussion in chapter 6 we may model the market price adjustment by including and modelling the purchasing rate of the customers/market in an competitive open-market setup. A well-known setup is hereby to structure $p_{i}(\cdot)$ in (13) by using the net present valuation approach of the insurance contracts. That is, to tune the premium $p_{i}(\cdot)$ in a way so the net present values of the contracts and the portfolio are optimized under some financial conditions (typically predefined financial plan goals). This approach takes care of what insurance business steering is all about, that is to find the optimal operational balance between running profitability and market share.

In addition to the claims risk process $Z(t)$, we assume the stochastic process of the purchasing rate $R(t)$ at time $t$. Let $Z_{i}(t)$ and $R_{i}(t)$ denote the claims risk and the purchasing rate for contract number $i$, and let $V_{i}(T)$ be the net present value of contract $i$ over the time period $(0, T)$. Hence we have:

$$
V_{i}(T)=\int_{0}^{T} e^{-r t}\left[p_{i}(t)-Z_{i}(t)-c_{i}(t)\right] R_{i}(t) d t,
$$

where

$R_{i}(0)=$ the hit sales rate of contract $i$ at time $t=0$, and

$R_{i}(t)_{t>0}=1-\widetilde{R}_{i}(t)_{t>0}$, where $R_{i}(t)_{t>0}=$ the renewal rate of contract $i$ at time $t$ and $\tilde{R}_{i}(t)_{t>0}=$ the cancellation rate of contract $i$ at time $t$. The practical interpretation of these definitions is that insurance customers are continuously purchasers through their continuously decisions of contractual cancellation or not.

In (14) remark also the assumed known constant parameters of $r=$ discount rate, and $c_{i}(t)=$ the total internal cost rate of contract $i$ at time $t$.

Hence the net present value $V(T)$ of the portfolio of all insurance contracts over the time period $(0, T)$ is

$$
V(T)=\sum_{i=1}^{I} V_{i}(T) \approx \int_{0}^{I} \int_{0}^{T} e^{-r t}\left[p_{i}(t)-Z_{i}(t)-c_{i}(t)\right] R_{i}(t) d t d i
$$

Algebraic - or more realistic, numerical - optimum solutions of (14) and (15) with respect to the net premium $p_{i}(\cdot)$ and preferable under some financial constraints, will implicitly generate the structure of the price model (13). The external market adjustments $M$ in (13) is hereby materialized through the stochastic purchasing variable $R$ in (14) and (15) which further on should be modelled by using the customer explanation parameters of for instance price sensitivity, price position in 
the market and soft preferences of product, service and/or branding, ref earlier discussions. Maybe also the distribution power of the insurer, which really has purchasing effect in most markets, should be included as an explanation variable. Hence, dependent of the purchasing preferences in the market, too high prices or too low prices both will generate lower net present values $V_{i}(T)$ and $V(T)$ than middle path price values. We should anyhow demand $E V(T)>0$ and $E V_{i}(T)>0$ for all $i=1, \ldots, I$, that is, we should have net sales prices which generate positive expected net present values of all insurance customers.

A challenge within this model is, however, to link this incomplete market setup with a local martingale approach based on market equilibrium, and hereby find explicit closed form expressions of the net premium $p_{i}(\cdot)$ in (13) as a function of the expected values of $E Z_{i}(T)$ and $E R_{i}(T)$, either under a real world probability measure $P$ or under an information filtered measure $Q$. We leave this challenging problem for future theoretical research.

One last comment before we finally end the insurance pricing approach by dealing with the parameter estimation: Sometimes the $E_{Q} N$ and $E_{Q} Y$ in (10) in the complete market case in chapter 5 are interpreted as the market price of claims frequency and market price of claims severity. This interpretation may be correct in a specific theoretical sense of view, but not necessarily in a general practical one. Within practical insurance pricing it is most often operational suitable to treat the classical pure risk premium on the physical risk measure $P$ apart from the other effects or elements of the pricing structure generated by some (individual) filtered measure $Q$. Hence in an operational setup we should split out, estimate and follow-up the real claims frequency and the real claims severity, instead of envelope them in some kind of imprecise and misleading market interpretation. Hence, all or most of the other effects of the insurance premium will turn out to be either the cost adjustment or the market adjustment of the risk price in (13), and hence easier to focus and follow-up explicitly.

\section{Parameter estimation $=$ real insurance pricing methodology}

The parameter estimation of the pricing models represents the real heart of insurance pricing. Compared to financial derivatives, the parameter estimation within non-life insurance is more complex. The main difference between the derivatives and the insurance contracts are hereby that derivatives are rather technical in their contractual consideration but quite simple in their parameter valuation, while the insurance contracts have the opposite characteristics. Hence while the volatility parameter of an option is most often estimated by practical market knowledge or implied real market volatility, the pure risk price pr risk in (13) is usually split into expected claim frequency and expected claim severity and hence estimated separately by using the data application machinery of classical statistical models like Generalized Linear (or Additive) regression (Mixed) Models (GLM, GAM or GLMM) or empirical Bayes credibility models on large data sets of risk exposure and reported claims. 
In chapter 3 we assumed the underlying claims number process $N(t)$ to follow a homogeneous Poisson process with constant frequency parameter $\lambda$, and hereby the claims risk process $X(t)$ to be a compound Poisson process. A far more common and realistic model is however to allow stochastic variation of the claims frequency from its expected value $\lambda$. Hence if $\lambda$ follows a Gamma distribution, then $N(t)$ becomes a Negative Binomial process. This setup matches very well our desire to make a risk differentiated premium tariff which reflects risk variances (both claims frequency and claims severities) between customers/objects in the insurance portfolio and market. Hence, this matches also the standardized GLM setup with Poisson/Gamma-assumptions. Important practical elements to handle within risk pricing are choice of explanation risk variables (tariff factors), estimation levels (product, coverage or claims type level), categorization of variables, correlations between variables, use of subjective experience/knowledge, follow up and maintenance. There is a wide number of references to pure risk pricing estimation techniques; an "old" classic is e.g. Brockman and Wright (1992), while a newer reference of applied GLM is Fahrmeir and Tutz (2000).

The purchasing rate $R(t)$ in (15) may also be modelled by using GLM. A well known and well used model is to assume the purchasing rate as a binary variable (the customer either purchase/renewal the contract at a discrete time, or he/she does not), and hence assume a Logistic Regression model with explanation variables based on the individual purchasing preferences of the customers, i. e. like pure customer characteristics, premium increase(s) last year(s) and the insurers price and distribution position in the market. Understanding the purchasing rates (both sales hit rates and renewal rates) for different customer segments - preferable by use of some stochastic models - should be a basic part of the model applications within pragmatic insurance pricing in incomplete markets.

Finally we stress the importance of forecasting the claims risk inflation or trend level. A sufficient prediction of the future trend level of the claims risk process $X(t)$ is extremely important within financial goal steering. The most pragmatic setup is to let the forecasting models be stand alone models apart from the GLM risk differentiation models, and hence influencing only the premium levels of the premium tariffs. However, using GLM-based leading indicators integrated with autoregressive integrated or moving average (ARIMA) models gives often an efficient modelling of the claims risk inflation.

\section{International best practice of insurance pricing}

A standard term of the pricing quality of the best insurance players in a market is best market practice or - when the pricing quality is among the best in the world world class practice. The international trend is a move from an internal cost based price focus to a market-oriented view on pricing, just like the wide approach in this paper. An increased number of consultants offer their knowledge and service to help the companies to reach a best practice level of pricing. Key elements of a best practice market-oriented view on pricing are: 
- Outstanding quality of the insurance claims risk and underwriting processes and their time dependent risk cycles. This quality is fundamental for profitable market based pricing.

- Outstanding knowledge of customer behaviour in terms of customer segments, products/service standards and price preferences, including own price and product/service positions relative to competitors in the market.

Hence not only the traditional actuarial pricing approach of risk and cost pricing, but also the business elements of CRM (Customer Relationship Management) including product and service standards should be important parts of the final premium tariff prices of insurance products and contracts. An example of a mix of these elements is e.g. treated in Holtan (2001), with focus on customer purchasing rationality of individual insurance bonus-malus contracts. This wide business approach demands definitely a dynamic pricing approach, that is, a process which continuously or regularly...

- adjust the price levels to reach risk ratio and combined ratio goals,

- innovate, update and re-estimate risk price models and parameters,

- track competitors' price rates and product/service value propositions,

- test market price adjustments to learn optimal price adjustments,

- implement champion-challenger price adjustments,

- communicate with distribution departments to optimize the sales forces.

This generates and assumes an active role of the analysts (actuaries) in the product and price departments of the insurance companies, with focus on dynamic processes as an overall operational key factor to succeed.

The methodology of risk and cost pricing - ref chapter 8 - is quite familiar to most insurance companies and markets. The methodology of market pricing is, however, far less familiar. It is important to recognize that there are no fixed rules or final answers to market price challenges and questions. A dynamic data driven (and less static theoretical model driven) working approach with flexible and short time-to-market IT price calculation systems seems, however, to be some kind of a common element of the best market price practicing insurance companies. Modelling the expected purchasing rate and the expected customer profitability in a net present value context, are hereby some key issues to handle.

References to best practice insurance pricing are in its nature quite few and difficult to put forward. The practice of Direct Line Insurance Ltd in UK - and their explosive way to reach $15-20 \%$ market share in the UK motor insurance market in the 1990-thies with satisfactory profitability - is often mentioned as an example. Other best players - that is, players with extremely good profitability in combination with stabile or growing markets shares over a significant period of time - are of course also good references. A readable journey - in spite of its age to best business focused risk/cost price and underwriting practice is however McKinsey (1995). 


\section{Summary and concluding remarks}

A fundamental difference between financial option valuation techniques and the classical actuarial valuation principles is that the option valuation is formulated within a market framework which includes the possibility of trading and hedging. This a priori model approach - which very much is consistent with the derivate market realities - generates in most cases complete market conditions without arbitrage possibilities. This proactive market model approach has traditionally been absent within classical actuarial pricing principles, whereas most of the principles have only been based on more or less ad hoc statistical considerations involving the law of large numbers. There seems however to be a theoretical trend to involve the financial market model approach into insurance pricing problems. Within such a framework there seems to be easier to expand the insurance price models by including components like purchasing preferences in incomplete market setups, and by this include not only the valuation part of pricing, but also the supply/demand part into the price models. A short overview of the pricing elements of insurance versus finance versus theory versus practice may be presented as follows:

\begin{tabular}{|c|l|l|}
\cline { 2 - 3 } Practise & $\begin{array}{l}\text { Risk and cost differentiated prices } \\
\text { Data driven GLM parameter estimation } \\
\text { Incomplete and non-efficient markets } \\
\text { Purchasing/price sensitive preferences } \\
\text { Data driven market adjusted net prices }\end{array}$ & $\begin{array}{l}\text { Still complete and efficient markets } \\
\text { Extreme contractual complexity } \\
\text { Different contractual objects } \\
\text { Computer formula based valuation } \\
\text { Implied volatility and market knowledge }\end{array}$ \\
\cline { 2 - 3 } Theory & $\begin{array}{l}\text { Insurance claims as underlying risk } \\
\text { Implicit complete markets } \\
\text { No hedging or arbitrage focus } \\
\text { Homo/heterogeneous Poisson processes } \\
\text { Risk valuation with safety loadings }\end{array}$ & $\begin{array}{l}\text { Stocks/securities as underlying risk } \\
\text { Explicit complete and efficient markets } \\
\text { Dynamic hedging with no arbitrage } \\
\text { Geometric Brownian motion processes } \\
\text { Volatility valuation and pricing }\end{array}$ \\
\cline { 2 - 2 } & \multicolumn{2}{|c|}{ Insurance } \\
\multicolumn{2}{|c}{ Finance }
\end{tabular}

The whole area of bringing together elements from financial and actuarial valuation and pricing techniques is still very much under construction. We should still expect a considerable progress with effects for insurance pricing in the years to come. Some future insurance challenges in the 21 st century with opposite directions are a) optimal pricing in incomplete friction markets with less price sensitivity (which is still the case for many or most traditional markets/customer segments) and b) optimal pricing in efficient and complete insurance markets with continuously insurance trading and hedging possibilities (future efficient internet and broker based markets; see Finnegan and Moffat (2000) for a pragmatic and problematic view on this subject). In the latter case the bridge to option pricing is very short. In both cases the pricing optimality should have a balanced focus on profitability versus market share of the (direct) insurance portfolio. In such an innovative framework actuaries in all countries should know how to act and take leading positions in their insurance companies to unite the future theoretical and practical parts of insurance pricing. 


\section{References}

Aase, K. K. (1993) Equilibrium in a reinsurance syndicate; Existence, uniqueness and characterization. ASTIN Bulletin 23, 185-211.

Black, F. and M. Scholes (1973) The pricing of options and corporate liabilities. Journal of Political Economy 81 (3), 637 - 654.

Brockman, M. J. and T. S. Wright (1992) Statistical Motor Rating: Making effective use of your data. Journal of Institute of Actuaries 119, 457-543.

Criss, N. A. (1997) Black-Scholes and Beyond - Option Pricing Models. McGraw-Hill.

Delbaen, F. and J. Haezendonck (1989) A martingale approach to premium calculation principles in an arbitrage free market. Insurance: Mathematics and Economics 8, 269 - 277.

Delbaen, F. and W. Schachermayer (1994) A general version of the fundamental theorem of asset pricing. Matematische Annalen 300, $463-520$.

Delbaen, F. and W. Schachermayer (1997) Non-Arbitrage and the fundamental theorem of asset pricing: Summary and main results. Proceedings of Symposia in Applied Mathematics, American Mathematical Society.

Embrechts, P. and S. Meister (1997) Pricing insurance derivatives, the case of CAT-future. Proceedings of the 1995 Bowles Symposium on Securization of Risk. Georgia State University, Atlanta. Society of Actuaries, Monograph M-FI97-1, 15-26.

Embrechts, P., R. Frey and H. Furrer (1999) Stochastic processes in insurance and finance. Working Paper, Department of Mathematics, ETH Zürich, Switzerland.

Embrechts, P. (2000) Actuarial versus financial pricing of insurance. Working Paper, Department of Mathematics, ETH Zürich, Switzerland.

Fahrmeir, L. and G. Tutz (2000) Multivariate Statistical Modelling Based on Generalized Linear Models. Second Edition. Springer.

Finn, J. and M. Lane (1997) The perfume of premium ... or pricing insurance derivatives. Proceedings of the 1995 Bowles Symposium on Securization of Risk, 27 - 35. Georgia State University, Atlanta. Society of Actuaries, Monograph MFI97-1, 27-35.

Finnegan, D. and S. Moffat (2000) Auto Insurance Pricing Crisis. Research report, Quality Planning Corporation, San Francisco, USA. 
Harrison, J. M. and D. M. Kreps (1979) Martingales and arbitrage in multiperiod securities markets. Journal of Economic Theory 20, 381 - 408.

Harrison, J. M. and S. R. Pliska (1981) Martingales and stochastic integrals in the theory of continuous trading. Stochastic Processes and their Applications 11, $215-260$.

Harrison, J. M. and S. R. Pliska (1983) A stochastic calculus model of continuous trading: Complete markets. Stochastic Processes and Applications 15, $313-316$.

Holtan, J. (2001) Optimal insurance coverage under bonus-malus contracts. ASTIN Bulletin 31, 179-190.

McKinsey (1995). The Journey. Working paper. McKinsey \& Co, USA.

Merton, R. (1973) The theory of rational option pricing. Bell Journal of Economics and Management 7, $141-183$.

Møller, T. (2000) Quadratic Hedging Approaches and Indifference Pricing in Insurance. $\mathrm{PhD}$ thesis, Laboratory of Actuarial Science, University of Copenhagen.

Sondermann, D. (1991) Reinsurance in arbitrage free markets. Insurance: Mathematics and Economics 10, 1991 - 202. 